\title{
Small Nucleolar RNA SNORA74A (U19)
}

National Cancer Institute

\section{Source}

National Cancer Institute. Small Nucleolar RNA SNORA74A (U19). NCI Thesaurus. Code C88996.

Small nucleolar RNA SNORA74A (U19) (184 bp) is encoded by the human SNORA74A gene. This small nucleolar RNA may play a role in both the conversion of uridine to pseudouridine and the processing of pre-ribosomal RNA. 